\section{AB0854 A CASE REPORT OF BROWN SYNDROME COMPLICATING THE MANAGEMENT OF SCLEROMYOSITIS}

P. Arora ${ }^{1}$, L. Croot ${ }^{1}{ }^{1}$ Barnsley Hospital NHS Foundation Trust, Rheumatology, Barnsley, United Kingdom

Background: Brown syndrome is a rare ocular motility disorder which has been reported in JRA, RA and SLE but never in a patient with scleromyositis.

Objectives: To report the first case of Brown syndrome in a patient with scleromyositis and increase awareness of this condition.

Methods: A case report and discussion.

Results: The patient was diagnosed with scleromyositis, at the age of 34 , after presenting with arthralgia, sclerodactyly, skin pigmentation, Raynaud's phenomenon, mild muscle weakness and dyspnoea. His labs were CRP $47 \mathrm{mg} / \mathrm{L}$, CK $868 \mathrm{IU} / \mathrm{L}$, ANA strongly positive; anticentromere Ab negative and Anti-PM/Scl-75 and Anti- PM/Scl-100 Ab positive. HRCT chest showed extensive pulmonary fibrosis with lower lobe honeycombing. TLCO was 3.98 (33\% of predicted).

He was initially managed with high dose steroids and pulsed IV cyclophosphamide with azathioprine for maintenance therapy. His lung disease stabilised and myositis resolved but he continued to develop calcinosis cutis so was switched to 6 monthly IV rituximab.

6 years later, he developed morning headaches with intermittent diplopia, described as double vision in vertical gaze with one image being above the other. Episodes lasting 10 minutes to 2 hours. Examination showed normal visual acuity and fundoscopy, no peripheral or eye muscle weakness.

Investigations to exclude myasthenia gravis, cerebral vasculitis and atypical infection were organised (MRI, AChR antibody, lumbar puncture, MRA) and were normal.

Because of intermittent nature of his episodes, his eye examination was always normal but he captured images in disconjugate gaze with right eye looking upwards and outwards when trying to look straight (Figure 1). Occasionally this was associated with orbital pain and an audible click. These features are suggestive of Brown syndrome.

He continues to have recurrent episodes despite immunosuppression but prednisolone $20 \mathrm{mg}$ daily for $1-2$ days at onset of each attack causes rapid resolution of symptoms.

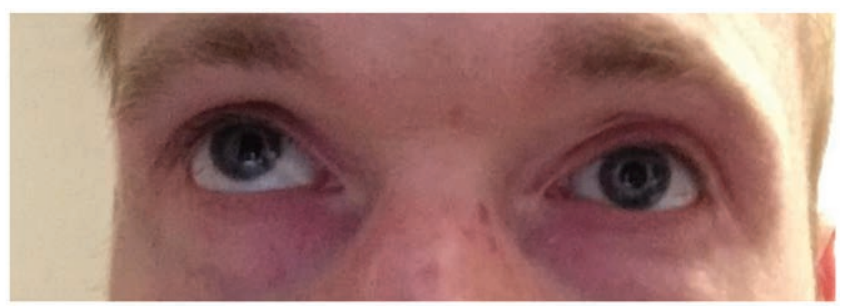

Figure 1. Right eye looking upwards and outwards when trying to look straight

Conclusion: Scleromyositis is an overlap syndrome of scleroderma and dermatomyositis. Muscle involvement is mild and clinical presentation can be variable. The PM/Scl antibodies are highly characteristic of the syndrome. (1)

Brown syndrome is an ocular motility disorder, first described in 1950, characterized by the inability to fully elevate the affected eye in adduction due to pathology of the superior oblique tendon sheath. (2)

It can be congenital or acquired, viz, trauma, surgery or sinusitis and also been described in RA, JIA and SLE. (3)

If superior oblique tendon cannot relax or slide freely through the trochlea then the affected eye cannot depress completely, leading to diplopia on upward gaze. (4) In inflammatory disease it is thought that swelling of the posterior part of the superior oblique tendon or tenosynovitis are likely causes of the tendon sheath abnormality. (4) This is likely to be the case in this patient because his symptoms are recurrent, respond to steroids and tend to occur more towards the end of rituximab cycles.

Recognition of this syndrome is important because invasive investigations can be avoided. Also, intermittent diplopia in a patient with autoimmune disease is suggestive of myasthenia gravis which maybe incorrectly diagnosed.

Finally, this case demonstrates the syndrome can be easily managed with short courses of oral steroids, although patients who are already on immunosuppressant treatment may need this in addition.

\section{REFERENCES:}

[1] Török L, Dankó K, Cserni G, Szûcs G. PM-SCL autoantibody positive scleroderma with polymyositis (mechanic's hand: clinical aid in the diagnosis) JEADV 2004; 18: 356-359

[2] Brown H W. Congenital structural muscle anomalies. In:Alien J H, ed. Strabismus ophthalmic symposium I. St Louis:CV Mosby, 1950: 205-6.

[3] Cooper C, Kirwan JR, McGill NW, Dieppe PA. Brown's syndrome: an unusua ocular complication of rheumatoid arthritis. Ann Rheum Dis 1990; 49:188-9.

[4] Sandford-Smith JH. Superior oblique tendon syndrome and its relationship to stenosing tenosynovitis. Br JOphthalmol 1973; 57:859-65.

Disclosure of Interests: None declared

DOI: 10.1136/annrheumdis-2021-eular.2454

\section{AB0855 FIBROUS ARTHROPATHY AS THE KEY FEATURE OF JUVENILE SCLERODERMA - CASE REPORT}

D. Dibrov ${ }^{1}$, M. Starovoytova ${ }^{1}$, O. Desinova ${ }^{1}{ }^{1}$ V.A. Nasonova Research Institute of Rheumatology, Department of Microcirculation and Inflammation, Moscow, Russian Federation

Background: Systemic sclerosis (SSc) is an autoimmune disease characterized by endothelial dysfunction, immunological disorders, and excessive synthesis of collagen and its deposition in various tissues and organs. The juvenile onset SSc before the age of 16 is very rare, with annual incidence of 0.27-0.5 cases per million children according to English and Finnish authors [1,2]

Objectives: To present a clinical case of juvenile onset SSc, manifesting from the childhood predominantly with fibrous contractures.

Methods: Patient K., 30 yo. Clinical presentation on admission to the Institute of Rheumatology in September 2020: thickening of the trunk and limbs skin (mRSS 10 scores), pronounced induration of subcutaneous tissues and muscles; contractures of the elbow, shoulder, hip and knee joints, short stature (height $142 \mathrm{~cm}$ ) with proportional shortening of the limbs. ANA (HEp-2) 1/320, a-Scl-70, a-RNP 70 and $A C A$ tests were negative. Ultrasonography revealed left-sided coxitis, esophagogastroduodenoscopy - Barrett's esophagus. Chest CT, echocardiography, electrocardiography and capillaroscopy yielded no specific findings.

The patient has been ill since the age of 3 , when SSc manifested with skin thickening, "dry" arthritis and rapid development of contractures of the large joints. Thorough diagnostic elaboration ruled out such potential causes as phenylketonuria, glycogenosis, mucopolysaccharidoses, primary amyloidosis, and porphyria. Histological findings (2007) of a biopsied skin specimen containing subcutaneous fat and muscle tissue included focal vacuolization of keratinocytes, poor perivascular lymphocytic and histiocytic infiltration, fibrosis and hyalinosis of collagen fibers of varying intensity in the in mid- and deep dermis, infiltration of collagen fibers by fibroblasts, skin appendages atrophy - all of them representing a pattern of morphological changes characteristic of SSc. Therapeutic regimens including prednisone at 5-15 mg/day and D-penicillamine were ineffective.

Results: In this case, in view of fibrotic arthropathy, a differential diagnosis was made with deep morphea and stiff skin syndrome. Visceral involvement, immunological disorders and biopsy findings substantiated a diagnosis of juvenile onset SSc. Oral MTX was initiated at $15 \mathrm{mg}$ to target skin lesion and osteoarticular symptoms

Conclusion: Predominance of fibrotic arthropathy in presented case caused difficulties in establishing SSc diagnosis, as this patient did not have such inherent features as the Raynaud's phenomenon, interstitial lung disease or pulmonary hypertension. Juvenile onset SSc manifesting before the age of 16 has its own clinical features, usually persisting through the adulthood, and therefore such one-of-a-kind appearances of juvenile onset SSc should not be missed or misinterpreted.

\section{REFERENCES:}

[1] Herrick AL, Ennis H, Bhushan M et al. Incidence of childhood linear scleroderma and systemic sclerosis in the UK and Ireland. Arthritis Care Res. 2010 Feb;62(2):213-8. doi: 10.1002/acr.20070.

[2] Pelkonen PM, Jalanko HJ, Lantto RK et al. Incidence of systemic connective tissue diseases in children: a nationwide prospective study in Finland. $J$ Rheumatol. 1994 Nov;21(11):2143-6

Disclosure of Interests: None declared

DOI: 10.1136/annrheumdis-2021-eular.2840

\section{$\mathrm{AB} 0856$ \\ CHRONIC INTESTINAL PSEUDO-OBSTRUCTION WITH HYDRONEPHROSIS: A CASE REPORT ON SUCH DISABLING AND RARE COMPLICATION OF LUPUS}

C. S. $\mathrm{Ng}^{1}$, S. L. Kan ${ }^{1}$, A. L. Lim ${ }^{1} .{ }^{1}$ Hospital Pulau Pinang, Rheumatology Unit, Medical Department, Georgetown, Malaysia 
Background: Chronic intestinal pseudo-obstruction can be a rare complication of systemic lupus erythematosus. It is often late to be identified in contrast to other commoner organs involvement in systemic lupus erythematosus such as nervous system, joint and kidney.

Objectives: To report a case of uncommon gastrointestinal complication of lupus, with associated hydronephrosis at the ureter, with treatment delay.

Methods: We report a case of chronic intestinal pseudo-obstruction.

Results: A 34 year old, with ten-year history of lupus nephritis, presented with recurrent abdominal pain and diarrhoea past nine months. The patient had just been diagnosed as end-stage renal failure a year ago, on regular haemodialysis. For the past ten years, the patient was not able to tolerate immunosuppressant due to the multiple episodes of infections, including shin carbuncle, herpes zoster, breast abscess and catheter-related candida/bacterial infections. However, the patient did not have any other major organs manifestations of lupus for the past ten years. The patient was apyretic. Multiple stool cultures were negative including Clostridium difficile. The abdominal radiography showed dilated small bowel with diffuse thickening of large and small bowels on computed tomography. Bilateral uretero-hydronephrosis was also noted without any evidence of obstructive uropathy on imaging. Ileocolic resection was done for presumed intestinal obstruction and the ileocolic biopsy did not reveal any granuloma, malignancy or vasculitis except for non-specific inflammation of cecum. Cytomegalovirus inclusion body was absent as well. Tuberculosis culture was negative. Oesophagoduodenoscopy and colonoscopy were offered in view of persistent unexplained loose stool and abdominal pain. But unremarkable findings were noted from multiple biopsy specimens of the small and large bowels. Second relook of the initial hemicolectomy specimen with special actin immunostain on the smooth muscle revealed degenerative changes of the muscularis propria. These were evidenced by cytoplasmic vacuolation, atrophy and pyknotic nucleus of the smooth muscle cells with surrounding oedema. Smooth muscle dysmotility could be the underlying pathology of this patient presentation. The patient responded well to intravenous immunoglobulin followed by azathioprine in addition to prednisolone and prokinetic agent.

Conclusion: Prompt recognition is pivotal in this case could have prevented the unnecessary surgical intervention earlier. It is potentially reversible. Long term prognosis of this rare entity is, however, varying.

Disclosure of Interests: None declared

DOI: 10.1136/annrheumdis-2021-eular.2854

\section{AB0857 CERTOLIZUMAB PEGOL RELOADING SUCCESSFULLY CONTROLS INFLAMMATORY ARTHRITIS FLARES DURING PREGNANCY - A CASE REPORT}

V. Pavlova ${ }^{1} .{ }^{1}$ McMaster University, Department of Medicine, Hamilton, Canada

Background: Approximately $50 \%$ of patients with inflammatory arthritis (IA) experience worsening of disease activity during pregnancy. ${ }^{1,2}$ Given the evidence that increased disease activity immediately before or during pregnancy is associated with adverse pregnancy outcomes, it is imperative to maintain tighter disease control during this period. ${ }^{3}$ Treatment options are limited to steroids or disease-modifying anti-rheumatic drugs (DMARDs) compatible with pregnancy. Certolizumab pegol (CZP) is a humanized TNF-alpha inhibitor with no to minimal transplacental transfer, making it a suitable option during pregnancy. ${ }^{4}$ In patients who relapse while on treatment with a biologic, consideration can be given to optimizing the dose of the biologic. Studies in Crohn's disease demonstrated that increasing the CZP dose from $400 \mathrm{mg}$ Q4W to Q2W is a viable strategy for recapturing efficacy. ${ }^{5}$ Similar data is not available for the rheumatology population or for pregnancy.

Objectives: To describe the successful control of juvenile inflammatory arthritis (JIA) flares using a reloading strategy with CZP in a pregnant patient with prior failure of multiple conventional systemic (cs)DMARDs and biologics.

Methods: This is a case report of a 31-year old female with JIA who presented with a disease flare in the $1^{\text {st }}$ trimester of her $2^{\text {nd }}$ pregnancy. The patient was treated with multiple csDMARDs and biologics since age 6. She experienced significant gastrointestinal side effects to subcutaneous methotrexate and leflunomide and inadequate response to hydroxychloroquine and sulfasalazine. Subsequently, she initiated and maintained treatment with etanercept for 14 years until she developed secondary loss of efficacy. She had primary non-response to golimumab and abatacept and experienced a severe allergic reaction to infliximab. Since 2017, she has been treated with CZP $200 \mathrm{mg}$ Q2W with good control of disease activity.

Prior to conception, the patient had low disease activity (Table 1). At 11 weeks gestation, the patient noted worsening of disease activity, with pain at 4/10, fatigue at $7 / 10$, morning stiffness for 60 minutes, swollen joint count (SJC)
4/28, and tender joint count (TJC) 19/28. The patient global assessment of disease activity (PtGA) remained at 4/10 while physician global assessment of disease activity (PhGA) and health assessment questionnaire disability index (HAQ-DI) increased to $6 / 10$ and 1.625 , respectively (Table 1). She reported that symptoms worsen one week after CZP injection with more joint pain and swelling in hands, wrists, and feet. The patient declined the addition of hydroxychloroquine, sulfasalazine, and steroids due to previous tolerability, lack of efficacy, and concerns about safety during pregnancy. The patient received 3 loading doses of CZP $400 \mathrm{mg}$ Q2W. She then continued treatment with CZP $200 \mathrm{mg}$ Q2W.

Table 1. Disease activity prior to conception and during pregnancy before and after reloading CZP.

\begin{tabular}{lccc}
\hline Characteristic & Prior to conception & 11 weeks gestation & $\begin{array}{c}19 \text { weeks gestation } \\
\text { after reloading CZP }\end{array}$ \\
\hline SJC (28 joints) & 2 & 4 & 2 \\
TJC (28 joints) & NA & 19 & NA \\
PhGA & 4 & 6 & 2 \\
PtGA $^{*}$ & 4 & 4 & 4 \\
HAQ-DI & 1.5 & 1.625 & 1.5 \\
Pain $^{*}$ & 4 & 4 & 2 \\
Fatigue $_{\text {Morning stiffness (min) }}$ & 7 & 7 & 5 \\
& 60 & 60 & $20-30$ \\
\hline
\end{tabular}

${ }^{\star}$ Assessed on a 10-point scale.

Results: On follow-up at 19 weeks gestation the patient reported $80 \%$ improvement in joint pain and swelling and reduction in morning stiffness (Table 1). Pain and fatigue were rated as $2 / 10$ and $5 / 10$, respectively. PtGA score was $4 / 10$, PhGA score was 2/10 and HAQ-DI score was 1.5 (Table 1).

Conclusion: Reloading CZP to control increased IA disease activity during pregnancy may be a viable treatment option. To our knowledge, this is the first report of dose optimization in IA and in pregnancy in a patient treated with CZP.

\section{REFERENCES:}

[1] van den Brandt S, et al. Arthritis Res Ther 2017;19(1):64.

[2] Jethwa, $\mathrm{H}$ et al, Arthritis Rheumatol. 2016;68 (suppl 10).

[3] de Man Y, et al. Curr Opin Rheumatol. 2014;26:329-333.

[4] Mariette X, et al. Ann Rheum Dis. 2018 Feb;77(2):228-233.

[5] Sandborn WJ, et al. American College of Gastroenterology Annual Scientific Meeting. 2009; Abstract 699

Disclosure of Interests: Viktoria Pavlova Speakers bureau: Amgen, Abbvie, BMS, Jenssen, Lilly, Merk, Novartis, Roche, UCB, Pfizer, Consultant of: Amgen, Abbvie, BMS, Jenssen, Lilly, Merk, Novartis, Roche, UCB, Pfizer, Grant/research support from: UCB

DOI: 10.1136/annrheumdis-2021-eular.3410

\section{\begin{tabular}{|l|l}
\hline AB0858 SUCCESSFUL TERM PREGNANCY IN A PATIENT \\
\hline
\end{tabular} WITH GRANULOMATOSIS WITH POLYANGIITIS AFTER UNDERGOING RITUXIMAB THERAPY - CASE REPORT}

O. Sirenko ${ }^{1}$, O. Kuryata ${ }^{1}$, T. Lysunets ${ }^{2}$, A. Legkobyt ${ }^{2}$, H. Mostova ${ }^{3}$.

${ }^{1}$ Dnipropetrovsk Medical Academy, Internal Medicine 2, Dnipro, Ukraine; ${ }^{2}$ Dnipropetrovsk Regional Clinical Hospital After Mechnicov, Rheumatology, Dnipro, Ukraine; ${ }^{3}$ Dnipropetrovsk Regional Clinical Hospital After Mechnicov, Obstetrics and Gynecology, Dnipro, Ukraine

Background: Granulomatosis with polyangiitis (GWP) is a relatively rare disease with the lack of experience in the management of pregnancy currently. Potentially negative factors of pregnancy prognosis are both disease-related and teratogenic effects of specific vasculitis therapy [1].

Objectives: To describe a clinical case of Successful term pregnancy in a patient with Granulomatosis with polyangiitis after undergoing rituximab therapy. Methods: 19 years-old woman was admitted to the rheumatology department of Clinical Regional Hospital After Mechnicov in 2013 with the debut of Granulomatosis with polyangiitis. Her disease course included recurrent rhinitis and sinusitis, Granulomatosis of the eye orbits. Positive ANCA titers were present in the disease onset. In the preceding 7 years she had been treated with corticosteroids, cyclophospan (discontinuated in 2017 because of lack of efficiency), than azathioprine. Aseptic necrosis of the both femoral head was estimated on fifth disease year. In 2018 azathioprine therapy had been discontinued owing to the disease progression and biological agents were prescribed - rituximab with positive effect. The disease remission was achived by rituximab therapy, the patient was warned about the need for contraception. The last dose of rituximab was introduced in February 2020. However, in March 2020, the patient reported pregnancy and therapy was discontinued. At the onset of pregnancy she was 